\title{
Phase Behavior of Dried - DDA Liposomal Formulation Dispersed in HPMC Matrix in the presence of Saccharides
}

\author{
Helmy Yusuf ${ }^{1 \star}$, Raditya Nugraheni ${ }^{1}$, Nur Aini Mulyadi ${ }^{1}$, Dwi Setyawan ${ }^{1}$, \\ Noorma Rosita ${ }^{1}$
}
Department of Pharmaceutics, Faculty of Pharmacy, Universitas Airlangga. Jl. Dharmawangsa Dalam, Surabaya 60286, Indonesia

\begin{abstract}
The present study describes the effect of saccharides and hydroxypropyl methylcellulose (HPMC) matrix on phase behavior of dehydrated cationic dimethyldioctadecylammonium (DDA)-based liposomes. Saccharides such as sucrose, lactose and mannitol,have been reportedpreserve the lipid membranes during drying, whilst HPMC matrix is widely used in solid dispersion to prevent aggregation and/or recrystallization. The study revealed that addition of sucrose and HPMCin the formulation demonstrated a miscible mixture that might construct a stable dried liposomal formulation. DTA data showed that sucrose (5\% w/v) and HPMC added to DDA liposomal formulation were relatively more miscible with the mixtures; whereas lactose and mannitol at the same concentration of 5\% showed phase separation from the mixtures in the dehydrated state. Furthermore, XRD and SEM analysis exhibited supporting evidences in which formulation using sucrose and lactose showedrelatively less crystalline-forming properties compared to formulation using mannitol. Recrystallization that cause phase separation might trigger leakage and further affect the efficacy of the entrapped drug/antigen. From these data, it might be concluded that a driedliposomal formulation can be prepared in the presence of sucrose (lyoprotectant) that is dispersed in HPMC matrix. The protective mechanism of sucrose $(5 \% \mathrm{w} / \mathrm{v})$ and HPMC matrix is proposed through inhibition of the recrystallization which causes phase separation; indicated by DTA, SEM and XRDdata. The present study revealed prospective advantages of using sucrose and HPMC in development of dried - DDA liposomal formulations.
\end{abstract}

Keywords : Liposomes, DDA, Sucrose, Lactose, Mannitol, HPMC, Phase Separation.

\section{Introduction}

The use of vaccines is considered as the most effective way to reduce or prevent the outbreak of epidemic of an infectious disease, so that vaccination is recommended rather than curative treatment in controlling the epidemic or even pandemic disease $\mathrm{e}^{1,2}$.

Most of available vaccines in the market is still formulated in liquid form and has many limitations. First, a liquid vaccine should be stored in a low temperature, range from $2-8^{\circ} \mathrm{C}$, and requires cold chain storage facilities (cold chain) during the distribution process ${ }^{3}$. Secondly, pain, discomfort and risk of spread of disease may increase in the use of syringes when the administration are not carried out properly. 
On the other hand, there are many advantages to be gained from dried vaccine. Those advantages include: the stability over temperature is much better; no need syringes; increased adherence to the immunization schedule; does not require special training for medical workers, increase the speed of distribution and reduce the cost of vaccination ${ }^{4}$.Therefore, the development of vaccine formulation in dry dosage forms to avoid the use of needles, is one of future perspectives to overcome these disadvantages.

Many studies in the field of vaccine have concluded that the use of the particulate system for vaccine delivery, such as liposomes and other nanoparticles can improve the effectiveness of antigens in inducing immune responses ${ }^{5,6}$. The interaction process of particulates with cells in mucosa (endocytosis) is more effective than without the use of particulates ${ }^{7}$.In this case, a successful delivery of antigen to mucosal tissue become an important factor in the effectiveness of vaccines ${ }^{8}$.

In this preliminary study, acationic lipid dimethyldioctadecylammonium (DDA); a cationic surfactant that also classified as quaternary(tetraalkyl) ammonium compound was selected to construct the liposome formulation. DDA has been reported to possess an adjuvant effect for decades ${ }^{9,10,11}$.It has been extensively used in vaccine studies and has been reported as an effective adjuvant for eliciting cell-mediated and humoural responses ${ }^{12,13}$. Furthermore,DDA liposomes have been used as vehicles for drug delivery or carrier systems for DNA $^{14,15,16}$.

However, the use of DDA in liposomal formulations is still challenging due to their stability

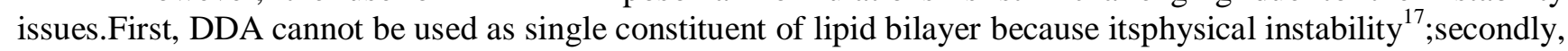
DDA vesicles tend to aggregate in pure water as the electrostatic compulsion is not sufficient to prevent their physical interactions ${ }^{18}$.

To overcome those problems, we applied strategies to enhance the stability including: addition of lyoprotectant i.e. saccharides (lactose, sucrose and mannitol) to stabilize the lipid membrane and also addition of a disperse matrix i.e. hydroxypropyl methylcellulose (HPMC)in the developed formulation. We focus on physical behaviorof those components during drying. Their behaviorin the driedform was charaterized based on their miscibility, phase separation and crystallinity. This phase behavior study of all components ina mixture as liposomal vaccine delivery systems may offer insight into development of potential new formulations of dehydrated liposomes as immunological adjuvant in vaccine formulations.

\section{Materials and Methods}

\section{Materials.}

Dimethyl-Dioctadecylammonium/DDA (Sigma Aldrich, Singapore), phospholipids/SPC (Lipoid $\mathrm{GmBh}$, Germany) were used as liposomal constituent, whilst cholesterol (Sigma-Aldrich, Singapore) were used as liposomal membrane stabilizer. Hydroxy-propyl-methyl-cellulose/HPMC (Shin-Etsu, Japan) was selected as a dispersion matrix, while sucrose, lactose or mannitol (Sigma-Aldrich, Singapura) was added to promote membrane stabilization upon drying and increase the mass for compact matrices of the resultant liposomes. Methanol (E. Merck) were selected as solvent to facilitate the mixing of liposomal ingredients. The solvent used was of analytical grade.

\section{Preparation of Dried Liposomes.}

Liposomes were prepared based on the thin film hydration technique. All of the prepared liposome formulations used in the current study are presented in Table 1. Briefly, all weighed amount drug and lipids were dissolved in methanol. Solvent was removed under vacuum for $2 \mathrm{~h}$. The lipid film was then hydrated with pre-warmed $\left(60^{\circ} \mathrm{C}\right)$ saccharide solution $(0,5 \% \mathrm{w} / \mathrm{v})$ in PBS buffer $(\mathrm{pH} 7.4)$ under consistent stirring for $15 \mathrm{~min}$. The formed liposomes were stored at room temperature for $0.5-1 \mathrm{~h}$ and sonicated using waterbath sonicator for $5 \mathrm{~min}$. A clearer dispersion should have been formed at this stage. The formed liposomes were then dispersed into a pre-formed HPMC gel and dried in oven at $40^{\circ} \mathrm{C}$ for more than $4 \mathrm{~h}$ to obtain solid product. All prepared formulations can be seen in Table 1 . 
Table 1.Formulations of Dried - DDA Liposomes

\begin{tabular}{|l|c|c|c|}
\hline \multirow{2}{*}{ Component } & \multicolumn{3}{|c|}{ Weighed Material (gram) } \\
\cline { 2 - 4 } & F1 & F2 & F3 \\
\hline DDA & 0.025 & 0.025 & 0.025 \\
\hline SPC & 0.025 & 0.025 & 0.025 \\
\hline Cholesterol & 0.005 & 0.005 & 0.005 \\
\hline Lactose & 0.5 & - & - \\
\hline Mannitol & - & 0.5 & - \\
\hline Sucrose & - & - & 0.5 \\
\hline HPMC & 0.25 & 0.25 & 0.25 \\
\hline
\end{tabular}

\section{Scanning Electron Microscopy (SEM).}

The morphology of the liposomes contained in solid gel was analyzed using SEM. The portions of the dried product were scattered and glued onto $25 \mathrm{~mm}$ diameter plates, which were attached to SEM specimen mounts. The specimens were sputter-coated with a layer of gold approximately $5 \mathrm{~nm}$ in thickness and specimens were examined with an electron microscope (TM 3000 Tabletop Microscope, Japan).

\section{Differential Thermal Analysis (DTA).}

DTA instrument (Mettler Toledo FP 85, Switzerland) was used to determine the phase transition temperature $(\mathrm{Tm})$ of the dried product. $\mathrm{Tm}$ was measured as endothermic peak minimum for the phase transition during the heating scan. The dry products were scanned from $30^{\circ} \mathrm{C}$ to $300^{\circ} \mathrm{C}$ at heating rate of $10^{\circ} \mathrm{C} / \mathrm{min}$. All samples were measured in three replicates using aluminum crucible.

\section{X-Ray Diffraction Analysis (XRD).}

Powder X-Ray Diffraction (Phillips X'Pert PRO PANalytical, Netherlands) analysis was performed at room temperature. Condition of measurement was set as follows: $\mathrm{Cu}$ metal target, $\mathrm{K} \alpha$ filter, voltage of $40 \mathrm{kV}$, $40 \mathrm{~mA}$. The analysis was performed on the range of 2 theta of $5-40^{\circ}$. Sample was placed on the sample holder and flatted to prevent particle orientation during preparation.

\section{Results and Discussions}

\section{Scanning Electron Microscopy (SEM)}

The morphology of dehydrated liposomes was analyzed by SEM. The SEM images showed the morphology of the dryproduct in the presence of saccharides (Figure 1). The dried sample had a relatively amorphous structure with smooth surface. Generally, such structure might be beneficial as it might lead to a faster reconstitution of the dry products. The SEM results confirmed that the use of saccharidesin the developed formulation and the drying method might able to maintain the miscibility of the mixtures after drying. A similar study using sucrose as protectant had been reported previously ${ }^{19}$.

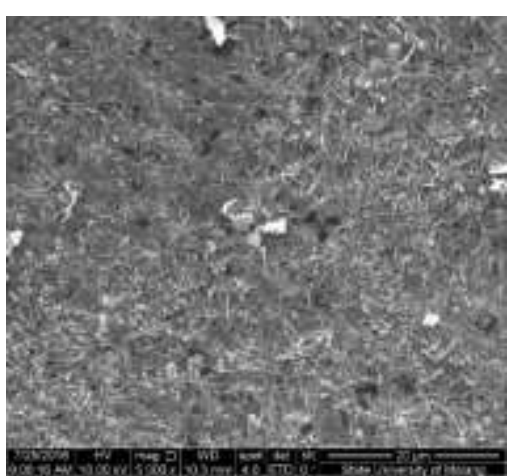

F1

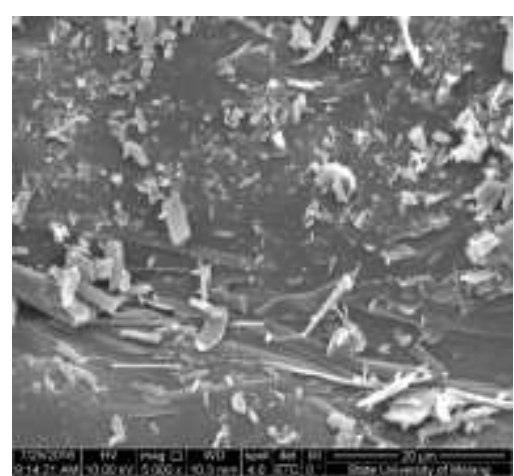

F2

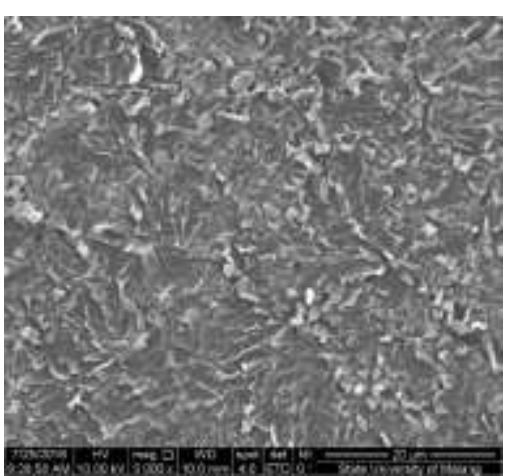

F3

Figure 1. SEM images of dried powder of each liposomalformulations, with magnification of 5000x. 


\section{Differential Thermal Analysis (DTA)}

Phase separation in liposomal membrane may have major disadvantage for their application as delivery systems. A phase separated component from the rest of the membrane will allow leakage of the encapsulated drug/antigen or even make the liposome collapse. Therefore, the effect of saccharides on the phase behavior (i.e. miscibility and phase separation) of dehydrated phospholipids mixtures was investigated. First, as can be seen from figure 2, we observed splitting peaks in the formulation containing sucrose $(\mathrm{F} 3)$ at $114,6^{\circ} \mathrm{C}$ and $205,6^{\circ} \mathrm{C}$. This sample exhibited slightly broad distribution of splitting without a clear discrimination. We suggested that the splitting endothermic peaks can be explained by the existence of sucrose-rich and sucrosepoor domains in the liquid-crystalline phase of the lipid bilayer; as similar case was found in a mixture of cholesterol at certain concentration with phospholipid ${ }^{20}$.This can also be concluded that sucrose at concentrations of $0,5 \% \mathrm{w} / \mathrm{vdid}$ not lead to phase separation during the dehydration process, indicating the good miscibility of all components.

Addition of lactose (F1) and mannitol (F2) in the mixture showed phase separation of components. The separated endothermic peak became much sharper and more obvious peaks for the use of lactose at $143,5^{\circ} \mathrm{C}$ and $204,3^{\circ} \mathrm{C}$; whilst observed at $144,1^{\circ} \mathrm{C}$ and $161,6^{\circ} \mathrm{C}$ when using mannitol(Figure 2). These results, therefore, imply that lactose and mannitol at concentrations of $0,5 \% \mathrm{w} / \mathrm{v}$ might lead to phase separation of the lipid mixture. We suggested that the same case applied to both formulation using lactose andmannitol. Their molecules tend to attract to other lactose or mannitol molecules rather than to those with the neighbouring lipid molecules and/or HPMC matrix and phase separated from the mixture.

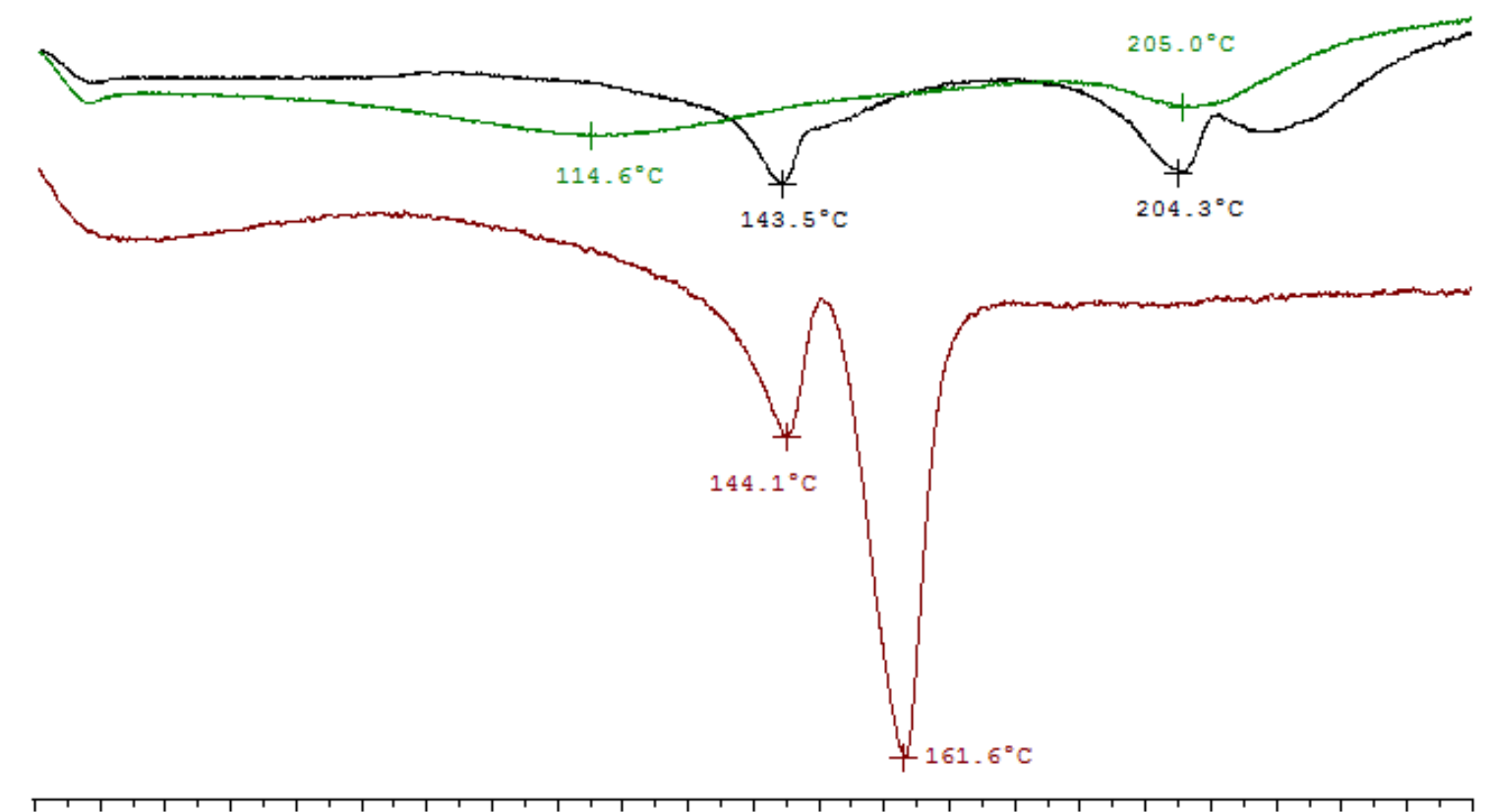

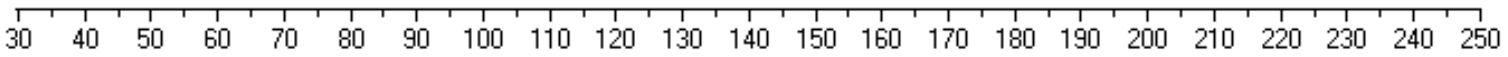

Figure 2. DTA thermogram of the dried powder of each liposomal formulations:F1 (Black), F2 (Brown) and F3 (Green).

It is suggested that the insertion of those saccharides between the lipid molecules decreased the strength of phospholipid packing in the gel phase; thereby reduces the rigidity of the membrane as the van der Waal's attraction between the acyl chains of phosphoplipids decreases. The rigidity of the liposomal membrane should be made sufficient in terms of not too fluid or firm. Very fluid membrane might leak out the encapsulated drug/antigen easily while too firm membrane decreases its permeability that makes it difficult for the encapsulated drug/antigen to pass through the membrane ${ }^{21,22}$. Therefore, in this case, we suggested that the concentration of sucrose $0,5 \% \mathrm{w} / \mathrm{v}$ fulfill the purpose of stabilizing liposomal membrane in the developed formulations. 


\section{X-Ray Diffraction Analysis (XRD)}

The X-ray diffraction patterns are presented in Figure3. Characteristically different diffraction patterns are obtained from each formula. For F1 (using lactose), the diffraction pattern displays a much less intens peaks, indicating a less crystalline-forming properties. On the other hand, F2 with mannitol and F3 with sucrose display a relatively more intens and sharper peaks that imply a more crytalline forming properties of the dry products. However, taking into account the SEM and DTA results that have been discussed previously, we suggested that the dry products exhibited partially crystalline and partially amorphous for F3, and fully crystalline for F2. The present of HPMC matrix was not able to act as barrier and prevent crystallization of lyoprotectant, led to phase separation of the mixture.

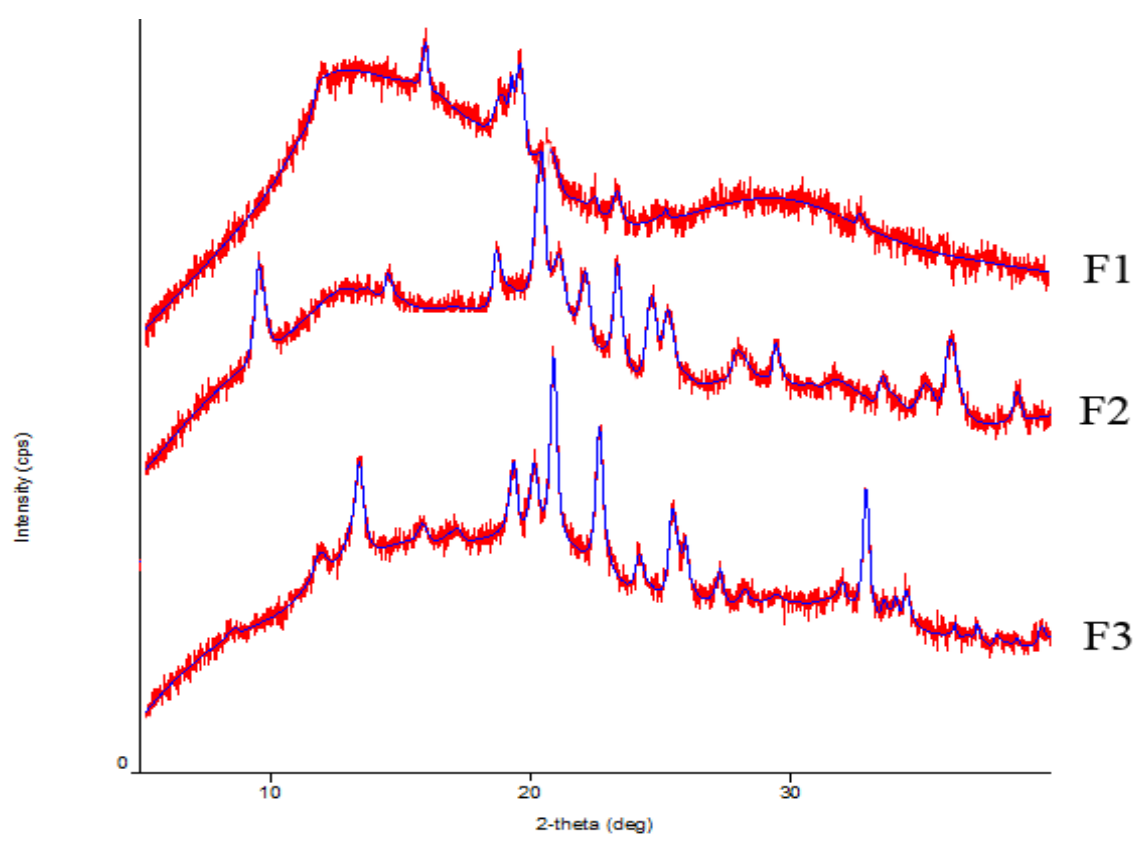

Figure 3. X-ray diffraction patterns of the dried powder of each liposomal formulations. F1, F2 and F3are the sample with lactose, mannitol and sucrose, respectively.

Saccharides, such as sucrose and lactose wereaddedto protect liposomal membranes from damage during drying ${ }^{23}$. One of the proposed mechanism is based on water replacement theory, in which sugar molecules are associated with polar head groups of dehydrated phospholipid bilayers by replacing the water molecules $^{24}$. Another mechanism is based on vitrification theory, in which the liposomes are protected from aggregation and fusion by entrapping them in avitrified matrix ${ }^{25}$. Mannitol has also been used as a protectant for liposomes. However, as can be seen from figure 3, the dry product showedmore cystalline-forming properties revealed by X-ray diffraction. The present of crytalls might disrupt the liposomes and cause leakage of the encapsulated drug/antigen, thus offer weaker protective effect. This results was in agreement with a reported study that mannitol do not interact with the lipid head groups, and only localize in the aqueous core ${ }^{26}$. The drying process was then causing the molecules to recrystallize and phase separated.

\section{Conclusions}

The present study demonstrated that dried - DDA liposomal formulation can be prepared in the presence of sucrose (lyoprotecant) that is dispersed in HPMC matrix.Concentration of sucrose $0,5 \% \mathrm{w} / \mathrm{v}$ applied to the mixtures ismiscible with all components in mixtures, while lactose and mannitol at the same concentration showed recrystallization andphase separated from the mixtures. The protective mechanism of sucrose as lyoprotectant and HPMC as dispersed matrix might be occurred through inhibition of the recrystallization which causes phase separation; indicated by DTA, SEM and XRDdata. 


\section{References}

1. R. Snacken, A.P. Kendal, L.R. Haaheim and J.M. Wood, Emerg. Infect. Dis., 5, 195 (1999); doi:10.3201/eid0502.990202.

2. R.J. Cox, K.A. Brokstad and P.L. Ogra, Scand. J. Immunol., 59, 1 (2004); doi:10.1111/j.03009475.2004.01382.x.

3. E. Weir and K. Hatch, CMAJ, 171, 1050 (2004); doi:10.1503/cmaj.1041565.

4. E.L. Giudice and J.D. Campbell, Adv. Drug Deliv. Rev., 58, 68 (2006); doi:10.1016/j.addr.2005.12.003.

5. M.F. Bachmann and G.T. Jennings, Nat. Rev. Immunol., 10, 787 (2010); doi:10.1038/nri2868.

6. $\quad$ N. Lycke, Nat. Rev. Immunol., 12, 592 (2012); doi:10.1038/nri3251.

7. M.J. Copland, T. Rades, N.M. Davies and M.A. Baird, Immunol. Cell Biol., 83, 97 (2005); doi:10.1111/j.1440-1711.2005.01315.x.

8. H.O. Alpar, S. Somavarapu, K.N. Atuah and V.W. Bramwell, Adv. Drug Deliv. Rev., 57, 411 (2005); doi:10.1016/j.addr.2004.09.004.

9. C.A. Kraaijeveld, H. Snippe, M. Harmsen and B.K. Boutahar-Trouw, Arch. Virol., 65, 211 (1980); doi:10.1007/BF01314537.

10. H. Snippe, L. Johannesen, E. Lizzio and B. Merchant, Immunology, 39, 399 (1980).

11. L.T. Hilgers and H. Snippe, Res. Immunol., 143, 494 (1992); doi:10.1016/0923-2494(92)80060-X.

12. L. Brandt, M. Elhay, I. Rosenkrands, E.B. Lindblad and P. Andersen, Infect. Immun., 68, 791 (2000); doi:10.1128/IAI.68.2.791-795.2000.

13. L. Holten-Andersen, T.M. Doherty, K.S. Korsholm and P. Andersen, Infect. Immun., 72, 1608 (2004); doi:10.1128/IAI.72.3.1608-1617.2004.

14. P.C. Barreleiro, R.P. May and B. Lindman, Faraday Discuss., 122, 191 (2003); doi:10.1039/B200796G.

15. G. Shi, W. Guo, S.M. Stephenson and R.J. Lee, J. Control. Release, 80, 309 (2002); doi:10.1016/S01683659(02)00017-2.

16. K. Lappalainen, A. Urtti, E. Söderling, I. Jääskeläinen, K. Syrjänen and S. Syrjänen, Biomembranes, 1196, 201 (1994); doi:10.1016/0005-2736(94)00224-X.

17. A. Vangala, D. Kirby, I. Rosenkrands, E.M. Agger, P. Andersen and Y. Perrie, J. Pharm. Pharmacol., 58, 787 (2006); doi:10.1211/jpp.58.6.0009.

18. J. Davidsen, I. Rosenkrands, D. Christensen, A. Vangala, D. Kirby, Y. Perrie, E.M. Agger and P. Andersen, Biomembranes, 1718, 22 (2005); doi:10.1016/j.bbamem.2005.10.011.

19. A. Wieber, T. Selzer and J. Kreuter, Eur. J. Pharm. Biopharm., 80, 358 (2012); doi:10.1016/j.ejpb.2011.11.008.

20. S. Ohtake, C. Schebor, S.P. Palecek and J.J. de Pablo, Biomembranes, 1713, 57 (2005); doi:10.1016/j.bbamem.2005.05.001.

21. K. Muramatsu, Y. Maitani, K. Takayama and T. Nagai, Drug Dev. Ind. Pharm., 25, 1099 (1999); doi:10.1081/DDC-100102275.

22. K. Inoue and T. Kitagawa, Biomembranes, 363, 361 (1974); doi:10.1016/0005-2736(74)90075-3. 
Helmy Yusuf et al/International Journal of PharmTech Research, 2017,10(1): 50-56.

23. J.H. Crowe, L.M. Crowe, J.F. Carpenter and C.A. Wistrom, Biochem. J., 242, 1 (1987); doi:10.1042/bj2420001.

24. P.T. Ingvarsson, M. Yang, H.M. Nielsen, J. Rantanen and C. Foged, Expert Opin. Drug Deliv., 8, 375 (2011); doi:10.1517/17425247.2011.553219.

25. W.L. Hinrichs, F.A. Mancenido, N.N. Sanders, K. Braeckmans, S.C. De Smedt, J. Demeester and H.W. Frijlink, Int. J. Pharm., 311, 237 (2006); doi:10.1016/j.ijpharm.2005.12.032.

26. G.M. El Maghraby, A.C. Williams and B.W. Barry, Int. J. Pharm., 292, 179 (2005); doi:10.1016/j.ijpharm.2004.11.037. 